\title{
Acute Pulmonary Embolism After Local Resection of Choroidal Melanoma
}

\author{
Weihong Yu
}

Choroid melanoma is a rare but primary ocular malignancy in adults, with an annual incidence of 6-7 cases per million people. Treatment options include radiotherapy (plaque brachytherapy, proton beam or stereotactic radiotherapy) and laser therapy. Transretinal endoresection using vitrectomy of choroidal tumors is performed only in a few centers because it requires special skills and hypotensive anesthesia, and also because of the concern of tumor seeding.

Beijing Tongren Eye Center reported three cases of lifethreatening complication of acute pulmonary embolism (APE) out of 682 cases of uveal melanoma local resection surgery during 1997-2011. All these three APE occurred within hours after surgery. The patients complained of discomfort, chest tightness, chest pain, and gasp with cyanosis. The BP dropped below 90/60 $\mathrm{mmHg}$, and ECG showed sinus tachycardia, lung-shaped $\mathrm{P}$ wave, and change in ST-T and SITIII. The blood gas analysis showed lowered $\mathrm{PaO}_{2}$, elevated $\mathrm{PaCO}_{2}$, and lowered $\mathrm{SaO}_{2}$. Ultrasonic cardiogram detected right ventricular enlargement. Chest X-ray showed pleural effusion. Two patients died and one patient received thoracentesis, low-molecular-weight heparin, and warfarin for anticoagulant therapy and eventually recovered.

The mechanism of emboli remains unknown. It is speculated that the emboli in these three cases may be derived from lower extremity venous thrombus. The first two patients, who died, received general anesthesia with controlled hypotension. The intraoperative BP was lowered to $75 / 40 \mathrm{mmHg}$ or $90 / 50 \mathrm{mmHg}$. The third patient received local anesthesia, and the BP was maintained normal during surgery. Since none of these two death cases underwent biopsy examination, other embolism sources including tumor tissue and silicone oil droplets could not be ruled out. Hypotensive general anesthesia reduced surgical bleeding, but it could also be a predisposing factor of thrombosis. The third patient received local anesthesia instead of general anesthesia; therefore it is also speculated that local resection surgery could possibly trigger the stress response and lead to embolism.

W. Yu $(\square)$

Key Laboratory of Ocular Fundus Diseases,

Chinese Academy of Medical Sciences \& Peking Union

Medical College, Beijing, China

Department of Ophthalmology, Peking Union Medical College

Hospital, Chinese Academy of Medical Sciences, Beijing, China 